Check for updates

1 Medical Sciences Division, University of Oxford, Oxford, UK

2 The BMJ, London, UK

Correspondence to: R Perera rafael.perera@phc.ox.ac.uk

Cite this as: BMJ 2021;373:n1159 http://dx.doi.org/10.1136/bmj.n1159 Published: 06 May 2021

\section{Thromboembolism and the Oxford-AstraZeneca vaccine}

\author{
New study finds a link, but vaccination remains overwhelmingly the safest option \\ Rafael Perera, ${ }^{1}$ John Fletcher $^{2}$
}

Medicine regulators, health professionals, and the public are anxious to know whether the available vaccines against covid-19 are safe, and in particular whether the Oxford-AstraZeneca vaccine causes unusual thromboembolic events. In a linked paper, Pottegård and colleagues (doi:10.1136/bmj.n1114) compared observed rates of vascular and thromboembolic events in large cohorts of adults who received the Oxford-AstraZeneca vaccine in Denmark and Norway, with expected rates derived from the general populations of the same countries. ${ }^{1}$

They found that for arterial events, the number of observed outcomes was similar to that of expected (83 observed events $v 86$ expected), but people given the vaccine experienced more venous thromboembolic events than expected (59 observed $v 30$ expected). Seven of these events were cerebral venous thrombosis, a life threatening condition identified in recent weeks as a potential complication of the Oxford-AstraZeneca vaccine. Seven cases among 281264 people vaccinated is a low absolute rate, but still 20 times the rate expected in the general population, and equivalent to an estimated 2.5 extra cases for every 100 ooo people vaccinated.

Faced with an association of this relative magnitude that appears to confirm a previous safety signal, prompts three important questions. Is this association likely to be real? How should we interpret Pottegård and colleagues' findings? What more do we need to know?

It seems quite likely that thromboembolic events were indeed more common among people who had the Oxford-AstraZeneca vaccine, but methodological uncertainties remain. As with all observational studies, critical steps must be taken to minimise potential bias. In this study, choice of an appropriate control group was one of the most difficult to manage.

Vaccinated participants were mostly health and social care workers, but Pottegård and colleagues used historical pre-pandemic population cohorts (from 2016 to 2019) to quantify baseline event rates-obtained after adjustment (standardisation) to match the age and sex profile of the vaccinated population.

Important differences exist between the vaccinated cohorts and the general population. For example, vaccinated cohorts are likely to be more healthy. At the same time, awareness of thromboembolic events might have been heightened during the pandemic, inflating reporting among the vaccinated cohorts relative to pre-pandemic controls. To address this issue, the authors did a separate analysis using control data collected during the pandemic, which showed similar results. Ultimately, a fully comparable control group is almost impossible in a study such as this, since important differences between vaccinated and unvaccinated cohorts will exist simply because of the selection process for vaccination.

The authors acknowledge that the lower than expected mortality observed in vaccinated cohorts is likely to be a consequence of selection bias, and they suggest that the association with venous thromboembolism may not be. It is a strength that this study was conducted specifically to investigate a possible association between vaccination and cerebral venous thrombosis-and found one.

The interpretation of Pottegård and colleagues' findings as either reassuring or concerning depends critically on the type of comparisons. While the rate of venous thromboembolism in vaccinated cohorts was higher than the background rate, we know that all vaccines against covid-19, including the Oxford-AstraZeneca vaccine, reduce mortality from covid-19 substantially. The absolute magnitude of this benefit varies with the proportion of people exposed to infection over time, but we do know from vaccine trials that mortality reduction far outweighs any risk of adverse events. We also know that covid-19 is itself associated with cerebral venous thrombosis-an estimated 4.3 events per 100000 infections, which is higher than the 2.5 per 100000 reported by Pottegård and colleagues. ${ }^{2}$

Comparing vaccine adverse event rates to background population rates is appropriate for rare diseases, since most people are never exposed to the disease but are exposed to risk of adverse events if vaccinated. The situation is different in a pandemic: most people are at risk of serious harm from infection, so comparisons with an unexposed population are less appropriate.

We now need to know whether the Oxford-AstraZeneca vaccine is unusual in its association with thromboembolic events, or whether other covid-19 vaccines share this characteristic. We also need to know how best to identify those at greatest risk if the association is real.

Even this large study observed too few adverse events for a definitive answer. While subgroup analyses hinted at particularly high risk of thromboembolic events among women younger than 45 , lack of a discernible effect in other groups, including men, might be due to the study's lack of power.

The choice we nearly all face is between eventual SARS-CoV-2 infection or vaccination. The Oxford-AstraZeneca vaccine is clearly a good choice, despite the likely risks found by Pottegård and colleagues. Quantifying the comparative risk associated with other vaccines is now a research (and public health) priority. 
The BMJ has judged that there are no disqualifying financial ties to commercial companies. The authors declare the following other interests: RP acknowledges part funding from the National Institute for Health Research (NIHR programme grant for applied research), NIHR Oxford Biomedical Research Centre, NIHR Oxford and Thames Valley Applied Research Collaborative (ARC), NIHR Oxford Medtech and In-Vitro Diagnostics Co-operative, and the Oxford Martin School.

Further details of The BMJ policy on financial interests is here: https://www.bmj.com/sites/default/files/attachments/resources/2016/03/16-current-bmj-education-coi-form.pdf.

Provenance and peer review: Commissioned; not externally peer reviewed.

1 Pottegård A, Lund LC, Karlstad Ø, etal. Arterial events, venous thromboembolism, thrombocytopenia, and bleeding after vaccination with Oxford-AstraZeneca ChAdOx1-S in Denmark and Norway: population based cohort study. BMJ 2021;373:n1114.

2 Taquet M, Husain M, Geddes JR, Luciano S, Harrison PJ. Cerebral venous thrombosis: a retrospective cohort study of 513284 confirmed COVID-19 cases and a comparison with 489 871 people receiving a COVID-19 mRNA vaccine. https://osf.io/a9jdq/ 